\title{
Diseño y desarrollo de proyectos STEM con equipos de escolares. Mentores universitarios para el proyecto Cambia Tu Mundo
}

\section{Susana Sastre-Merino ${ }^{a}$, Iciar Pablo-Lerchundi ${ }^{\mathrm{b}}$ y Javier Rey Rodríguez}

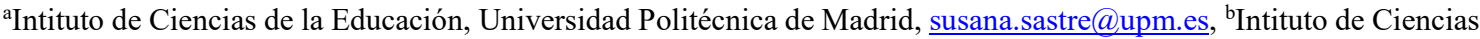
de la Educación, Universidad Politécnica de Madrid, iciar.depablo@upm.es y ${ }^{c}$ Universidad Politécnica de Madrid, javier.reyro@alumnos.upm.es.

\section{\$EWWDW}

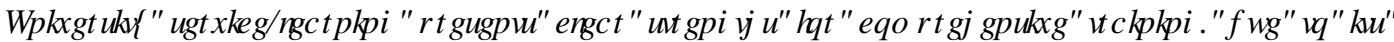

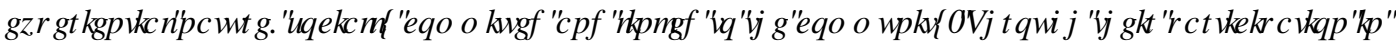

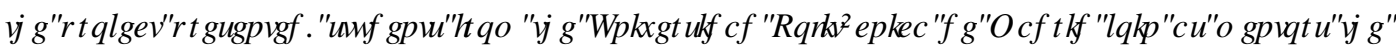

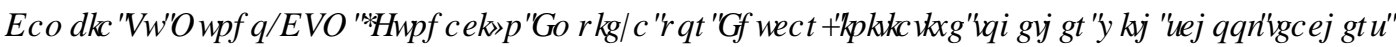

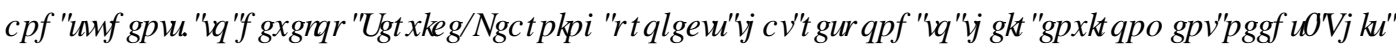

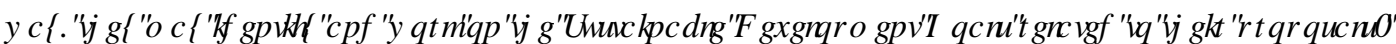

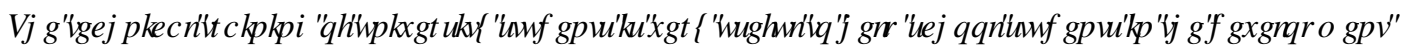

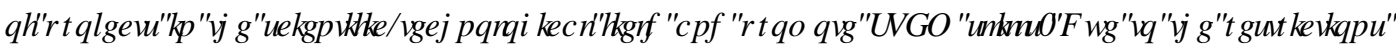

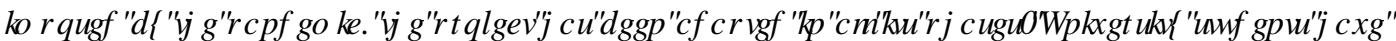

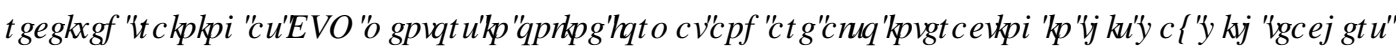



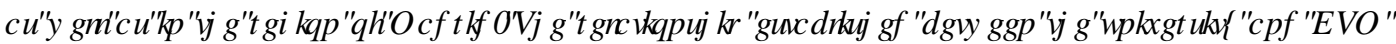

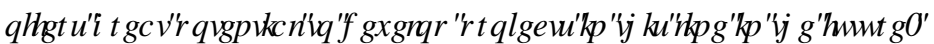

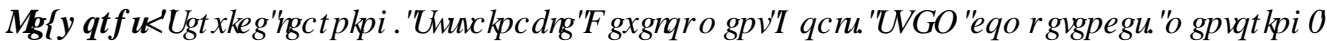

[]

\section{HXP HQ}

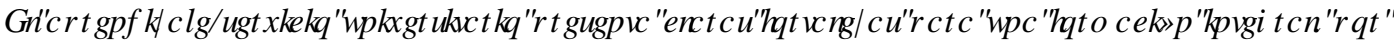

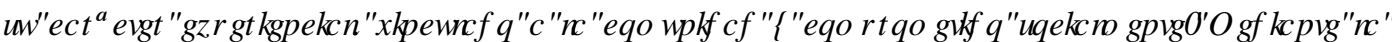

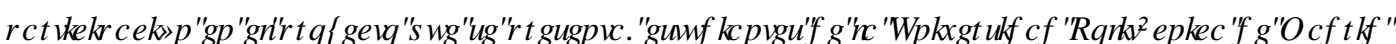

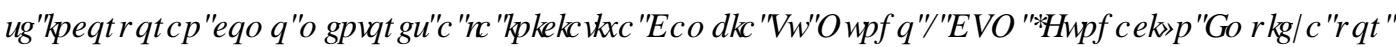

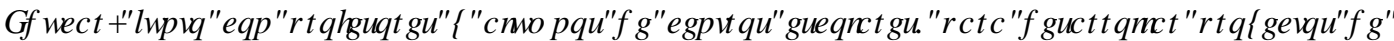

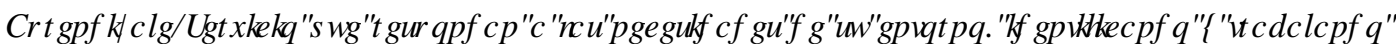

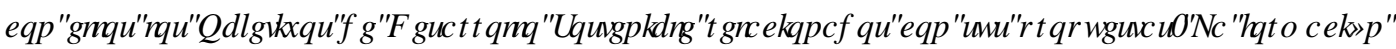

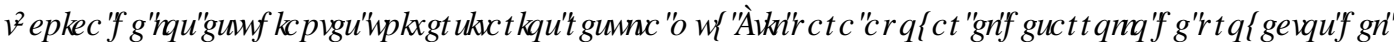

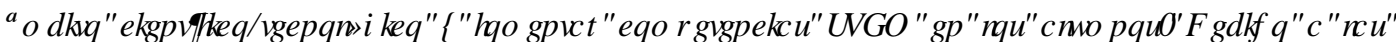

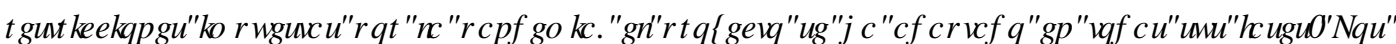

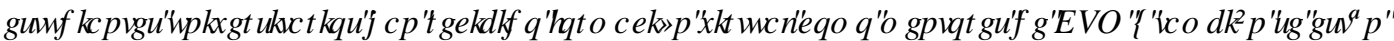

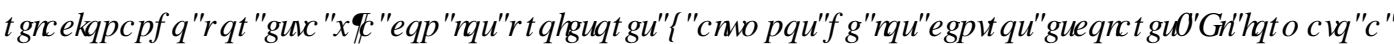

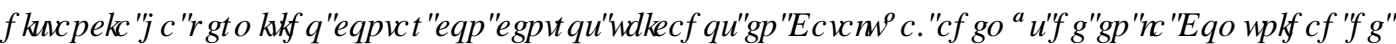

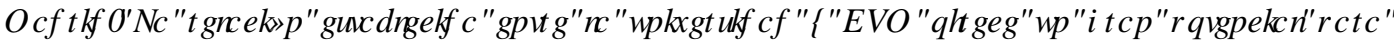

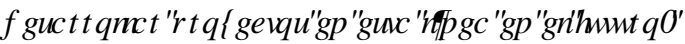

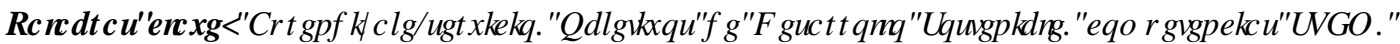
P HQURLDDW 


\section{Introducción}

El Aprendizaje-Servicio (ApS) en el contexto universitario presenta un enorme potencial para que los alumnos interactúen con la sociedad, realizando un servicio solidario a la vez que aprenden y desarrollan competencias técnicas, transversales, sociales y éticas que serán de gran valor en su desarrollo profesional y personal (Bringle et al., 2019; López-de-Arana Prado et al., 2020, Smith-Tolken \& McKay, 2019). Como afirma Rodríguez Gallego (2014), presenta ventajas en el currículum académico, al promover una formación práctica en un contexto aplicado, en la formación en valores, contribuyendo a la formación para la ciudadanía y en su vinculación con la comunidad, promoviendo la resolución de demandas y necesidades reales. Incluir proyectos de aprendizaje-servicio en la educación superior implica otrogar al estudiante un rol activo, de protagonismo, lo cual ofrece numerosas ventajas como el aumento de la motivación, una mayor conciencia social, fomento del pensamiento crítico etc., sin dejar de lado el rendimiento académico, que también se ve beneficiado (Novak, Markey \& Allen, 2007).

Además, se postula como un método que contribuye al desarrollo de los Objetivos de Desarrollo Sostenible (ODS), que forman parte de su propio núcleo (García Laso et al. 2019). Concretamente, la experiencia que se está llevando a cabo y que se presenta en esta comunicación enfoca su acción en el desarrollo del ODS 4, tratando de contribuir a una educación de calidad; y en el ODS 17, generando alianzas estratégicas con otros actores del ámbito educativo.

Por otro lado, existe una clara demanda social de fomentar competencias científico-tecnológicas en el alumnado de todos los niveles educativos para contar con generaciones que se adapten bien a lo que el futuro profesional y social les va a exigir. Recibir una formación relacionada con las competencias STEM (por las iniciales en inglés de Ciencia, Tecnlogía, Ingeniería y Matemáticas) resulta prioritario en una sociedad con un fuerte enfoque hacia lo tecnológico y digital, donde la mayoría de los empleos del futuro aún no se han inventado (Shatunova et al., 2019). Las empresas demandan trabajadores con una sólida preparación en competencias STEM (Conde et al., 2021) siendo el grado de formación y experiencia en el ámbito científico-tecnológico, incluso, un indicador de desarrollo de un país (Frolov, 2010). También la contribución al crecimiento económico de empresas de nueva creación, se ha relacionado con su base científico-tecnológica y la formación STEM de los emprendedores (Morales-Alonso et al., 2016). En definitiva, fomentar competencias científico-tecnológicas en los niños y jóvenes resulta crucial para el desarrollo de nuestra sociedad, por lo que hay que buscar metodologías y actividades para incluirlas en los currícula académicos de manera motivante para el alumnado. Sin duda, una aproximación desde el aprendizaje-servicio puede resultar una buena manera de involucrar a estudiantes universitarios y, a su vez, atraer a alumnos de Educación Secundaria hacia el ámbito STEM y entrenar sus competencias científicotecnológicas.

Teniendo en cuenta lo anterior, la Universidad Politécnica de Madrid (UPM), una de las grandes referentes nacionales en formación STEM, lleva años promocionando proyectos de innovación educativa en la línea del ApS en ámbitos muy variados como la agricultura, la eficiencia energética, el espacio urbano, el transporte etc. Desde 2017, junto con el resto de universidades públicas madrileñas, tiene un convenio con el Ayuntamiento de Madrid para promover el ApS. Adicionalmente, en el curso 2019-2020 se abrió la Oficina de Aprendizaje-Servicio (http://aprendizajeservicio.upm.es/), cuya misión se centra en fomentar el aprendizaje a través de la experiencia práctica que facilita esta metodología. Ofrece apoyo a docentes a través de convocatorias, formación o ayuda en la gestión de proyectos y difusión de resultados y a 
estudiantes, quienes pueden participar en proyectos de Aprendizaje-Servicio y recibir créditos académicos con ellos.

En este contexto, aunando el servicio a la comunidad con una formación de calidad de estudiantes en el ámbito STEM, se está llevando a cabo el proyecto que aquí se describe, para formar a estudiantes de la UPM como mentores de alumnos de centros escolares en formación de competencias científicotecnológicas y que éstos desarrollen distintos proyectos que repercutan en su entorno más cercano. Para ello, este proyecto de ApS incluye la colaboración de la UPM con la Fundación Empieza por Educar (ExE), a través de su proyecto Cambia Tu Mundo (CTM). ExE es una fundación independiente y sin ánimo de lucro comprometida con la promoción de la equidad educativa, trabajando en Madrid, Cataluña y País Vasco para que todos los estudiantes, empezando por los más vulnerables, tengan, gracias a la educación, vidas logradas. Forma parte de la red internacional Teach for All (www.teachforall.org) que engloba más de 50 organizaciones de diferentes países.

El proyecto Cambia Tu Mundo (https://empiezaporeducar.org/cambia-tu-mundo/) es una iniciativa coordinada por ExE, que busca desarrollar en el alumnado un sentimiento de ciudadanía y empoderamiento social para que estos puedan liderar proyectos sociales. Esto se logra a través de la metodología de aprendizaje-servicio, mediante la cual los estudiantes son protagonistas de su propio aprendizaje para fomentar la confianza en sí mismos y en su capacidad de liderar proyectos que mejoren el mundo en el que viven. El proyecto está dirigido tanto a alumnos de primaria como secundaria, incluyendo formación profesional y bachillerato.

En concreto, CTM consiste en una serie de talleres que acompañan y guían al profesorado y sus estudiantes para poder implementar el Aprendizaje-Servicio en el aula y para conocer los ODS. CTM ofrece dos itinerarios alternativos para abarcar las distintas asignaturas del nivel escolar: Itinerario General y STEM. El último ofrece talleres adicionales a los profesores para iniciarse en programación y robótica, así como en diseño de páginas web y apps, favoreciendo así las vocaciones STEM en los escolares.

El proyecto se lleva a cabo desde hace ocho años de manera paralela en varios centros de Madrid y Barcelona, habiendo participado alrededor de 5.500 estudiantes con más de 300 proyectos. Durante el curso escolar 2018-2019, se implantó además de forma piloto en Euskadi (en varios centros de Bizkaia y Araba).

En este contexto, y concretamente en el itinerario STEM, a lo largo de las ediciones previas, muchos profesores señalaban que a pesar de la formación que reciben del proyecto a través de los talleres, a menudo les resultaba difícil responder a las necesidades tecnológicas concretas que plantean los alumnos en muchos de los proyectos, bien por falta de medios o de tiempo, por lo que desde CTM se planteó la posibilidad de reforzar las formaciones y lanzar un programa de mentores. Sin embargo, no contaban con los voluntarios necesarios para llevarlo a cabo. Como respuesta a lo anterior, se planteó la alianza con los estudiantes de la UPM. Además del apoyo a los profesores y alumnos con sus proyectos, el programa de mentores se pensó como elemento de conexión de los escolares con su posible futuro, con una mirada externa y alternativa a la habitual.

Así en el curso 2019-2020, se solicitó desde el Instituto de Ciencias de la Educación (ICE) un proyecto de ApS en la convocatoria promovida por la Oficina de Aprendizaje Servicio de la UPM, para crear un programa de mentores universitarios. El proyecto CTM en ese curso contaba con 25 centros escolares y 1.222 alumnos a nivel nacional, Para la puesta en marcha de la alianza del proyecto con la UPM se planteó que los mentores UPM pudieran apoyar a los centros situados en la Comunidad de Madrid, que eran 11 centros y 826 alumnos. Sin embargo, el confinamiento de la población en marzo de 2020 por el COVID19 impidió avanzar en la convocatoria de los mentores y se tuvo que aplazar al siguiente curso. El proyecto CTM sí pudo completarse en la mayoría de los centros, para lo cual se aplicó un plan de contingencia para

(cc)) BY-NC-ND 2021, Universitat Politècnica de València

CRQJUHR, Q5 HGHपिए० 
poder continuar con las formaciones de manera digital. De esta manera, se mantuvieron involucrados 35 profesores y 1.063 alumnos celebrando los aprendizajes del curso en un evento final virtual. Así lo relata una profesora participante, la cual explica que "HOSLRJUP DUHDODSWUU SIGDP HQMADXQHQURLQRIRQOQHI I

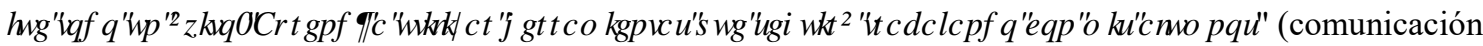
personal).

Durante el curso 2020-2021, se retomó el proyecto, realizando una adaptación de todo el diseño para responder a las restricciones impuestas por la pandemia. Se ofertó como actividad acreditable de 2 ECTS (correspondientes a una dedicación de 55 horas) la mentoría de proyectos STEM en centros escolares de Madrid y Cataluña. La actividad se ofreció a todos los alumnos de grado de la universidad dentro de las actividades acreditables por la UPM, en la acción "S.2. Actividad solidaria y de cooperación". Se admitió a 30 estudiantes.

Para la formación y seguimiento de los mentores, se cuenta con un equipo de docentes de la UPM, entre los que hay expertos en mentoría, así como especialistas en la formación en STEM para el ámbito de educación secundaria. Los docentes apoyan a los mentores en todas las dudas que puedan tener para el desarrollo de los proyectos en los centros escolares. Por parte de ExE, se cuenta con un equipo de cinco personas coordinando el proyecto CTM, de las cuales dos forman el equipo de relación con la universidad y los mentores. Este equipo está apoyado por un estudiante del Máster de Formación del Profesorado de ESO, Bachillerato y Formación Profesional de la UPM.

En cuanto a la participación en el proyecto CTM, en la presente edición participan siete centros de la Comunidad de Madrid y ocho en Cataluña, lo que hace un total de 15 centros. Los cursos son muy variados, desde $4^{\circ}$ Primaria hasta $2^{\circ}$ Bachillerato, incluyendo Formación Profesional Básica. El número de alumnos implicados en el proyecto es de 816 (585 de la Comunidad de Madrid y 231 de Cataluña).

\section{Objetivos}

Los objetivos que se plantean para este proyecto de innovación educativa son:

- Desarrollar competencias transversales como la comunicación, creatividad, resolución de problemas, a partir de un servicio real prestado a centros escolares.

- Aplicar conocimientos técnicos a la resolución de problemas reales planteados con los escolares, lo que permite trascender el enfoque técnico y abordar aspectos sociales y solidarios y relacionarlos con los ODS, que completan su formación como ingenieros. De esta forma, los alumnos UPM verán cómo sus conocimientos pueden ser útiles para la mejora de la sociedad.

- Servir de apoyo útil a escolares y a sus profesores, pues abre la mirada a posibilidades de resolución de problemas que no contemplarían probablemente con los recursos a su alcance. De ello resulta el enriquecimiento de los proyectos, elevando su calidad, y de los participantes, que encuentran posibles referentes futuros y los anima a su vez a trabajar para construir una sociedad mejor.

- Colaborar en un proyecto escolar de ApS que no entra en competencia con el trabajo que podría realizar una entidad profesional, al realizarse en el contexto educativo, con recursos limitados. La organización de CTM no tiene presupuesto disponible para contratar mentores profesionales que apoyen a los equipos de forma continuada.

- Mejorar la motivación de los alumnos al aplicar sus conocimientos en contextos reales.

- Fomentar las alianzas entre universidad - sociedad civil - educación escolar, para contribuir al cumplimiento del ODS 17. 
- Promover el despertar de vocaciones STEM en los estudiantes de primaria y secundaria (ESO, FP básica y Bachillerato).

\section{Desarrollo de la innovación}

Para el desarrollo del proyecto de innovación, se definieron una serie de fases (Figura 1).

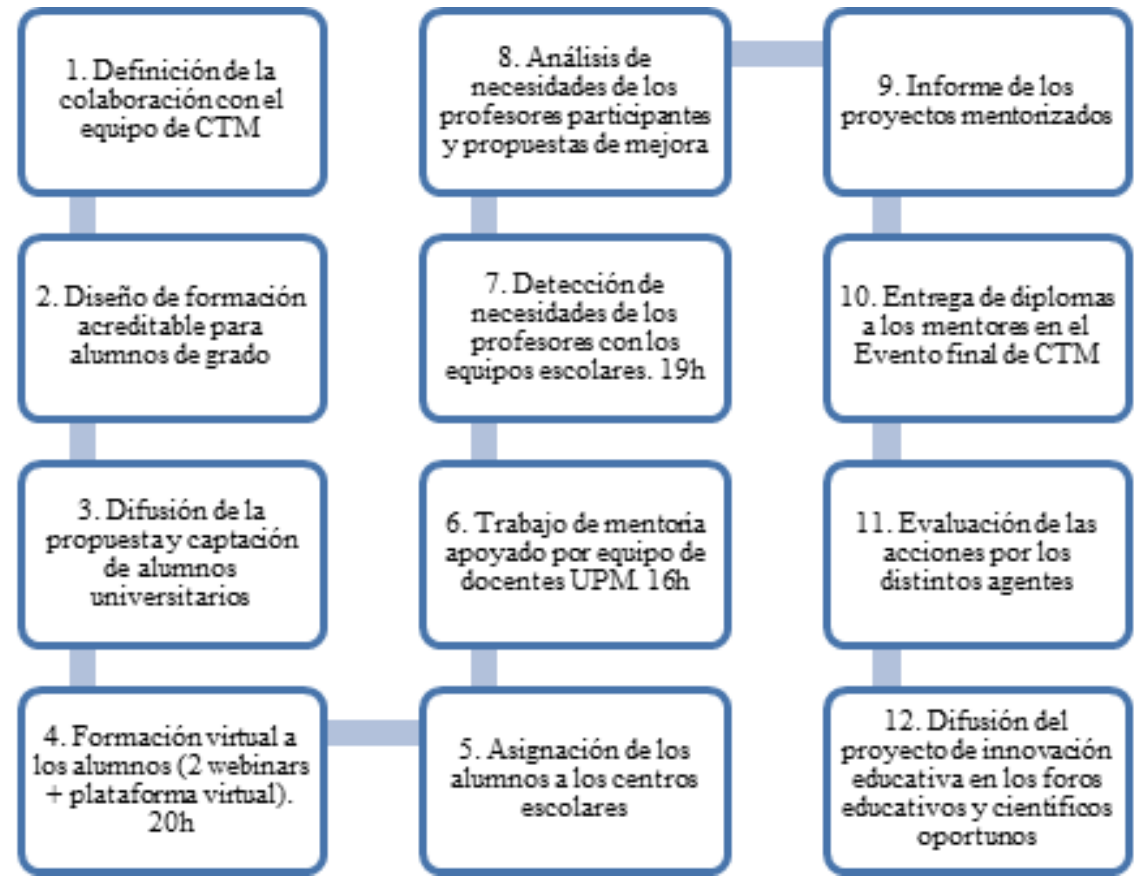

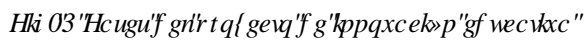

El equipo de trabajo universidad - organización de la sociedad civil (UPM-CTM) comenzó a diseñar el proyecto durante el curso 2019-2020. Tras posponerlo por la pandemia, se reestructuró la actividad acreditable para poder ofrecer la formación de manera virtual y no presencial, y para redefinir la relación de los mentores con los profesores y alumnos de los centros escolares. Se diseñaron dos escenarios, de total virtualidad y de posibilidad de que los mentores pudieran acudir a los centros. Esto último finalmente no ha sido posible.

Tras la difusión de la actividad y la inscripción de los interesados, se comenzó el proyecto en los meses de noviembre y diciembre de 2020. En los dos webinars se realizó una introducción al proyecto CTM y se dieron nociones básicas para el acompañamiento y mentorización al profesorado y alumnado. El primer webinar se realizó de forma exclusiva para los mentores UPM y el segundo fue compartido con el profesorado de los centros. A través de la plataforma de formación virtual, disponible también para el profesorado participante en CTM, se ofrecieron tres módulos de formación para realizar de manera individual y autónoma (Aprendizaje y Servicio, Design Thinking y Herramientas STEM). Además, se especificaron las funciones de los mentores y los próximos pasos para comenzar las mentorías.

Los mentores fueron asignados a los centros y comenzaron el contacto con los profesores. Debido a la pandemia, los plazos de cada fase se tuvieron que adecuar constantemente, y si bien los profesores pueden realizar sus proyectos con los alumnos desde principios de año, la mayoría de ellos acabó desarrollando sus proyectos en el tercer trimestre del curso. Por ello, los mentores tuvieron la oportunidad de acompañar a 
los grupos desde la fase de búsqueda conjunta de la necesidad a la que dar solución. La metodología de trabajo en los centros es la de Aprendizaje Basado en Proyectos. Los mentores pudieron participar en distintos momentos del desarrollo de los proyectos, dependiendo de lo indicado por los profesores asignados. Cada profesor establecerá las condiciones para la relación con los mentores. En algunos casos podría ser posible la conexión directa online con la clase, y en otros, la relación sería sólo entre mentor y profesor, dadas las distintas organizaciones logísticas realizadas por los centros para la adaptación de la docencia a las restricciones (que van desde la presencialidad diaria de toda la clase en dos turnos, a la rotación semanal de la mitad de la clase con conexión online).

La participación de los mentores finaliza con la entrega de un informe en el que reflejen un resumen de los proyectos mentorizados y del aprendizaje realizado y un análisis de las posibles necesidades de formación de los profesores en el aula para desarrollar los proyectos, así como propuestas de mejoras para las siguientes ediciones. De esta forma, los mentores realizan también un servicio a la propia organización de CTM, aportando una información muy valiosa para el futuro del proyecto.

Su participación será reconocida en el evento final del proyecto, que a diferencia de ediciones anteriores, en las que se reunían todos los centros participantes en una jornada de celebración, será virtual en esta edición y se celebrará el 11 de junio.

El seguimiento y evaluación del proyecto se realiza por parte del equipo UPM-CTM, apoyado por un estudiante del Máster de Formación del Profesorado de ESO, Bachillerato y Formación Profesional de la UPM. El seguimiento de los mentores se realiza semanalmente, para detectar cualquier incidencia en la relación con los profesores y alumnos. Además, una vez definidos los temas de los proyectos ApS seleccionados, los mentores podían contar con el apoyo de un tutor entre los profesores de la UPM involucrados en el proyecto, que les asesorarían en aquellos aspectos técnicos que así lo requieran. Los mentores serán evaluados teniendo en cuenta diferentes aspectos:

- Asistencia a las formaciones.

- Seguimiento por parte del equipo UPM y CTM.

- Cuestionario de evaluación de la actuación del mentor por parte de los profesores receptores del servicio y del equipo CTM.

- Informe de necesidades de los profesores participantes en CTM detectadas y propuestas de mejora.

- Informe de los proyectos mentorizados y aprendizajes realizados con la participación en la actividad.

Asimismo, se diseñaron diferentes herramientas de evaluación dirigidas a todos los agentes involucrados en el proyecto, para valorar globalmente la experiencia de colaboración entre la Universidad Politécnica de Madrid y la fundación ExE.

Además, el equipo del proyecto elaborará un compendio de los proyectos mentorizados, así como de las necesidades de los profesores participantes y propuestas de mejora realizadas por los mentores. También se dará difusión a la experiencia una vez terminada, en los foros educativos y científicos oportunos.

\section{Resultados}

En primer lugar se analiza la participación de los estudiantes universitarios en el proyecto. La Figura 2 muestra los perfiles académicos de los estudiantes que se inscribieron en la actividad acreditable. Se observa que predominan claramente los alumnos de informática y telecomunicaciones. 


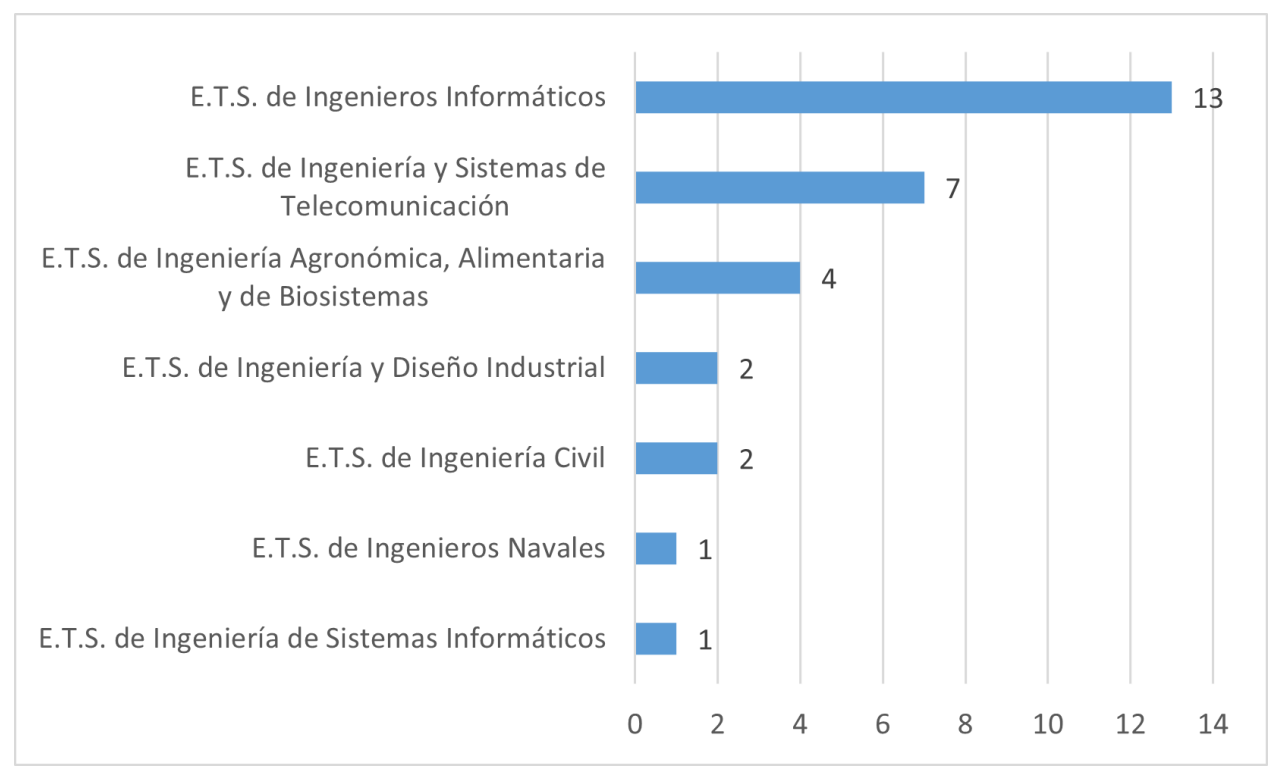

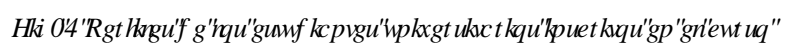

En la parte formativa del proyecto (Aprendizaje), de los 30 inscritos, 29 realizaron la primera formación. Los que realizaron las dos formaciones virtuales completas fueron 22. A continuación, estos se dieron de alta en la plataforma de formación virtual del proyecto CTM para poder completar su formación.

En relación con la parte de Servicio, los 22 alumnos que completaron la formación fueron asignados a los centros educativos participantes. Después de la asignación, cuatro alumnos se dieron de baja de la actividad por diferentes circunstancias, como haber dado positivo en COVID o haber suspendido asignaturas del primer semestre, y se tuvo que realizar una reasignación para que todos los profesores inscritos en el proyecto tuviesen un mentor asignado.

Como se observa en la Tabla 1, los 18 mentores fueron asignados a distintos profesores, repartidos en 15 centros, en los que atienden a un total de 29 profesores que participan con sus clases en CTM. Uno de los mentores (mentor número 11) se encarga de dos centros y el resto de uno. En el caso de los centros con más de un profesor participante, en algunas ocasiones se asignaron varios profesores a un mismo mentor.

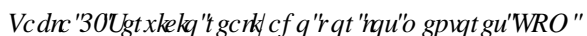

\begin{tabular}{|c|c|c|c|c|c|c|c|}
\hline $\begin{array}{l}\text { Mentor } \\
\text { UPM }\end{array}$ & $\begin{array}{l}\text { Centro } \\
\text { asignado } \\
\text { (provincia) }\end{array}$ & Curso & $\begin{array}{l}\mathrm{N}^{0} \text { de } \\
\text { alumnos }\end{array}$ & $\begin{array}{l}\text { Mentor } \\
\text { UPM }\end{array}$ & $\begin{array}{l}\text { Centro } \\
\text { asignado } \\
\text { (provincia) }\end{array}$ & Curso & $\begin{array}{l}N^{0} \quad \text { de } \\
\text { alumnos }\end{array}$ \\
\hline 1 & $\begin{array}{lr}\text { Colegio } & \text { Nuestra } \\
\text { Señora } & \text { de } \\
\text { Fátima (Madrid) }\end{array}$ & $2^{\circ} \mathrm{ESO}$ & 30 & 11 & $\begin{array}{l}\text { Salesianos } \\
\text { Estrecho } \\
\text { (Madrid) }\end{array}$ & $2^{\circ} \mathrm{ESO}$ & 100 \\
\hline 2 & $\begin{array}{l}\text { Colegio Vedruna } \\
\text { (Madrid) }\end{array}$ & Tutoría & 17 & 11 & $\begin{array}{l}\text { FEDAC } \\
\text { Manresa } \\
\text { (Barcelona) }\end{array}$ & $2^{\circ}$ Bach. & 20 \\
\hline 3 & $\begin{array}{l}\text { Colegio Vedruna } \\
\text { (Madrid) }\end{array}$ & $\begin{array}{l}4^{\circ} \quad \text { Ed. } \\
\text { Primaria }\end{array}$ & 20 & 12 & $\begin{array}{l}\text { Institut Baix a } \\
\text { Mar (Barcelona) }\end{array}$ & $\begin{array}{l}3^{\circ} \text { y } 4^{\circ} \\
\text { ESO }\end{array}$ & 20 \\
\hline 4 & $\begin{array}{l}\text { Colegio Vedruna } \\
\text { (Madrid) }\end{array}$ & $\begin{array}{l}5^{\circ} \quad \text { Ed. } \\
\text { Primaria }\end{array}$ & 58 & 13 & $\begin{array}{l}\text { Institut de } \\
\text { Terrassa } \\
\text { (Barcelona) }\end{array}$ & $4^{\circ} \mathrm{ESO}$ & 56 \\
\hline 5 & $\begin{array}{l}\text { Colegio Vedruna } \\
\text { (Madrid) }\end{array}$ & $\begin{array}{l}4^{\circ} \text { y } 5^{\circ} \mathrm{Ed} . \\
\text { Primaria }\end{array}$ & 20 & 14 & $\begin{array}{l}\text { Institut Pau } \\
\text { Claris } \\
\text { (Barcelona) }\end{array}$ & $1^{\circ}$ Bach. & 15 \\
\hline 6 & Colegio Vedruna & $2^{\circ} \mathrm{ESO}$ & 100 & 15 & Escola & $2^{\circ} \mathrm{ESO}$ & 50 \\
\hline
\end{tabular}




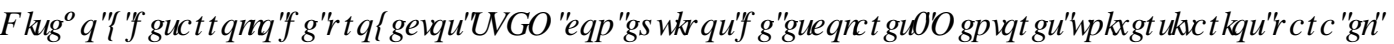
SLR HFIRI\&DP EID] 7 XIO XQCR]

\begin{tabular}{|c|c|c|c|c|c|c|c|}
\hline & (Madrid) & & & & $\begin{array}{l}\text { Martinet } \\
\text { (Barcelona) }\end{array}$ & & \\
\hline 7 & $\begin{array}{l}\text { Colegio Rafaela } \\
\text { Ybarra (Madrid) }\end{array}$ & $3^{\circ} \mathrm{ESO}$ & 90 & 16 & $\begin{array}{l}\text { Centre Escolar } \\
\text { San Francisco } \\
\text { (Barcelona) }\end{array}$ & $\begin{array}{l}3^{\circ} \text { y } 4^{\circ} \\
\text { ESO }\end{array}$ & \\
\hline 8 & $\begin{array}{l}\text { Institución La } \\
\text { Salle (Madrid) }\end{array}$ & $2^{\circ} \mathrm{FPB}$ & 35 & 17 & $\begin{array}{l}\text { FEDAC Salt } \\
\text { (Girona) }\end{array}$ & $4^{\circ} \mathrm{ESO}$ & 20 \\
\hline 9 & $\begin{array}{l}\text { Nuestra Señora } \\
\text { de Montserrat } \\
\text { (Madrid) }\end{array}$ & $2^{\circ} \mathrm{ESO}$ & 30 & 18 & $\begin{array}{l}\text { Institut Maria } \\
\text { Rúbies (Lleida) }\end{array}$ & $\begin{array}{l}4^{\circ} \quad \text { ESO } \\
y \quad 1 \quad \text { o } \\
\text { Bach. }\end{array}$ & 30 \\
\hline 10 & $\begin{array}{l}\text { Colegio Divino } \\
\text { Corazón } \\
\text { (Madrid) }\end{array}$ & $4^{\circ} \mathrm{ESO}$ & 6 & & & & \\
\hline
\end{tabular}

Durante el mes de febrero, los mentores tuvieron los primeros contactos con los profesores. El 100\% de ellos contactó con sus profesores asignados presentándose y ofreciendo su ayuda aunque en casi todos los casos, tuvieron que mantenerse a la espera de que los profesores comenzasen con sus proyectos en el tercer trimestre para poder realizar la mentoría. Sólo algunos mentores iniciaron en febrero-marzo reuniones con los profesores para conocer más en profundidad los proyectos, así como para especificar los ODS con los que están vinculados dichos proyectos.

Debido a las dificultades del curso, dos centros se dieron de baja del proyecto CTM. Uno de los mentores siguió apoyando a otro centro asignado, y el otro trató de ser reubicado, aunque no pudo finalmente establecer la colaboración con el profesor. Además, varios profesores sí continuaron participando en CTM pero no solicitaron ayuda alguna a los mentores.

En la tabla 2 se muestran los títulos de los proyectos definidos en los centros. Todos los proyectos están vinculados a uno o varios Objetivos de Desarrollo Sostenible y a temas de actualidad y preocupación de los alumnos escolares.

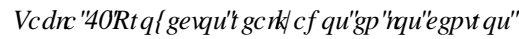

\begin{tabular}{|c|c|c|}
\hline $\begin{array}{l}\text { Mentor y Centro } \\
\text { asignado } \\
\text { (provincia) }\end{array}$ & $\begin{array}{l}\text { Proyectos } \\
\text { presentados a CTM }\end{array}$ & Descripción \\
\hline $\begin{array}{l}\text { 1.- Colegio } \\
\text { Nuestra Señora de } \\
\text { Fátima (Madrid) }\end{array}$ & $\begin{array}{l}\text { 1.-Aplicación para } \\
\text { elegir carrera (ODS } \\
\text { 4) } \\
\text { 2.-Lámpara } \\
\text { gravitatoria (ODS 7) }\end{array}$ & $\begin{array}{l}\text { 1.-Mediante AppInventor hacer una aplicación en la que, } \\
\text { introduciendo tus gustos y habilidades, te recomienda entre las } \\
\text { diferentes carreras y FP. } \\
\text { 2.-Elaborar lámparas que funcionen con la fuerza de la gravedad. }\end{array}$ \\
\hline $\begin{array}{l}\text { 2.- Colegio } \\
\text { Vedruna (Madrid) }\end{array}$ & - & El profesor dejó el proyecto. \\
\hline $\begin{array}{l}\text { 3.- Colegio } \\
\text { Vedruna (Madrid) }\end{array}$ & - & El profesor dejó el proyecto. \\
\hline $\begin{array}{l}\text { 4.- Colegio } \\
\text { Vedruna (Madrid) }\end{array}$ & $\begin{array}{l}\text { Ciudad sostenible } \\
\text { (ODS } 11 \text { y } 13)\end{array}$ & $\begin{array}{l}\text { Diseñar edificios sostenibles y fomentar transportes no } \\
\text { contaminantes. }\end{array}$ \\
\hline $\begin{array}{l}\text { 5.- Colegio } \\
\text { Vedruna (Madrid) }\end{array}$ & $\begin{array}{l}\text { Ciudad sostenible } \\
\text { (ODS } 11 \text { y } 13)\end{array}$ & $\begin{array}{l}\text { Diseñar edificios sostenibles y fomentar transportes no } \\
\text { contaminantes. }\end{array}$ \\
\hline $\begin{array}{l}\text { 6.- Colegio } \\
\text { Vedruna (Madrid) }\end{array}$ & $\begin{array}{l}\text { 1.-Apaga las luces } \\
\text { por el plantea (ODS } \\
\text { 7) } \\
\text { 2.-Conéctate por la } \\
\text { educación (ODS 4) }\end{array}$ & $\begin{array}{l}\text { 1.-Crear una serie de escenarios para concienciar a la población } \\
\text { sobre energías renovables. } \\
\text { 2.-Diseñar una manera de comunicación entre alumnos de distintos } \\
\text { colegios. } \\
\text { 3.- Concienciar a los niños pequeños de que no hay que tirar la }\end{array}$ \\
\hline
\end{tabular}


3.- Bob Esponja salva la vida submarina (ODS 14)

4.- Por un agua más limpia (ODS 6)

5.- Igualdad (ODS 5)

7.- Colegio

Rafaela Ybarra (Madrid)

Salle (Madrid)

9.- Nuestra Señora de Montserrat (Madrid)

\section{0.- Colegio}

Divino Corazón

(Madrid)

11.- Salesianos Estrecho (Madrid)
8.- Institución La

Los ODS y su uso para mejorar tu entorno (ODS 3, 4, 5, 7 y 14 )

El bichote (ODS 14)

Protocolo Covid (ODS 3)

1.-\&RZ₫\%[ (ODS 3)

2.-3IDQRTI* DPH

(ODS 4)

3.- HDQUKR+ HS

(ODS 4 y 13)

4.-4 XDOUFV(ODS 12

y 15$)$

5.-6FDSH\&RYIG(ODS

$3,13$ y 15$)$

6.- Corregato (ODS 3 y 4)

7.-3 HW) RXQAHV 9ILWDO' IDU (ODS

$8,9,15$ y 17)

8.-7DP DJRFKII

DQP DOR(ODS 4)

9.-5 HD IQJ ISDFH

(ODS 3)

10.-0 HP RUUDD H

(ODS 4)

11.-9LMRQH

(ODS 3, 7, 8 y 9)

\section{1.- FEDAC}

Manresa

(Barcelona)

12.- Institut Baix a Mar (Barcelona)

1.- Valla inteligente (ODS 11)

2.-Semáforo antiCovid19 (ODS 11)

13.- Institut de

Terrassa

(Barcelona)
1.-Aforo limitado

(ODS 3)

2.-Alarma de acoso basura mediante un programa diseñado en Scratch y un vídeo en Powtoon.

4.- Juego de Scratch para concienciar de forma divertida sobre la importancia de hacer buen uso del agua como bien escaso.

5.- Con el programa de Scratch se pone el ejemplo de una

mujer que no se siente segura a su alrededor y que su problema se soluciona llamando al 016 para que la policía sepa su ubicación y puedan ayudarla.

Reflexiones en equipos de cuatro, plasmadas en presentaciones con PowerPoint, sobre la forma en la que los ODS intervienen para mejorar su entorno.

Crear un robot capaz de andar por el fondo marino, detectar basura y recogerla.

El centro dejó el proyecto.

Crear un aparato mediante Arduino que mida la distancia entre mesas para que emita un pitido cuando no se respete la distancia de seguridad.

1.-Controlar el número de lavados de la mascarilla de tela.

2.-Enseñar a niños de entre 6 y 10 años a tocar el piano con las teclas básicas.

3.-Unir a las familias y concienciar de la importancia del reciclaje y cuidado del medio ambiente.

4.-Reutilizar el agua previamente purificada, para el consumo de animales y plantas.

5.-Crear un videojuego para escapar del Covid para concienciar de las medidas de seguridad para reducir contagios.

6.-Búsqueda del bienestar de nuestros gatos.

7.-3HW) RXQQHU Evitar la pérdida de tu mascota.

9IUWDO' IDU: conseguir que las personas desorganizadas tengan una mayor organización.

8.-Dar consejos de cómo cuidar a determinados animales domésticos, incluyendo en la aplicación un animal virtual. 9.-Reducir el estrés en niños y adolescentes entre 8 y 16 años mediante la comedia.

10.-Ayudar a las personas con problemas de memoria mediante el entrenamiento neuronal con un videojuego.

11.-Ayudar a las personas invidentes mediante un sensor infrarrojo que detecta el movimiento y objetos o personas.

El centro dejó el proyecto.

1.-Habilitar el uso del huerto del centro mediante sensores incorporados en la valla.

2.-Diseñar un semáforo para los pasillos que señale si es recomendable circular por ellos o no.

1.- Cuando el sensor detecte el máximo de aforo emitirá un sonido y una luz.

2.-Existencia de un botón por las calles con una cámara que se 


\begin{tabular}{|c|c|c|}
\hline & $\begin{array}{l}\text { (ODS 5) } \\
\text { 3.-Jugar a mejorar el } \\
\text { mundo (ODS 13) } \\
\text { 4.-Mejora tu } \\
\text { alimentación (ODS } 2 \\
\text { y 3) } \\
\text { 5.-Persiana } \\
\text { automática con LDR } \\
\text { (ODS 3) } \\
\text { 6.-Matemáticas } \\
\text { interactivas (ODS 4) } \\
\text { 7.-Mejorar el } \\
\text { reciclaje (ODS 11, } 12 \\
\text { y 13) }\end{array}$ & $\begin{array}{l}\text { activa cuando se pulsa el botón. } \\
\text { 3.-Reducir la contaminación otorgando recompensas a la gente } \\
\text { mediante distintos retos. } \\
\text { 4.-Informar a la gente sobre los alimentos que les conviene comer } \\
\text { para mejorar su salud y bienestar. } \\
\text { 5.-Consiste en una persiana que se regula automáticamente en } \\
\text { función de la hora del día y la luz de incide del exterior. } \\
\text { 6.-Aprender matemáticas de una forma más divertida. } \\
\text { 7.-Acabar con los residuos contaminantes y hacer que la gente sea } \\
\text { responsable a la hora de reciclar mediante Arduino y sensores en } \\
\text { los contenedores. }\end{array}$ \\
\hline $\begin{array}{l}\text { 14.- Institut Pau } \\
\text { Claris (Barcelona) }\end{array}$ & $\begin{array}{l}\text { Analizador de } \mathrm{CO} 2 \\
\text { en el aire (ODS } 11 \text { y } \\
13)\end{array}$ & $\begin{array}{l}\text { Controlar la concentración de } \mathrm{CO} 2 \text { en el aula mediante placas } \\
\text { Arduino y sensores } \mathrm{CO} 2 \text { y determinar cuando es necesario ventilar } \\
\text { el aula. }\end{array}$ \\
\hline $\begin{array}{l}\text { 15.- Escola El } \\
\text { Martinet } \\
\text { (Barcelona) }\end{array}$ & $\begin{array}{l}\text { Las plantas que lloran } \\
\text { (ODS } 13 \text { y } 15)\end{array}$ & $\begin{array}{l}\text { Montaje de un sensor de humedad y un dispositivo de audio con un } \\
\text { kit de Arduino, en una planta, para emitir un sonido al detectar } \\
\text { niveles bajos de agua y así avisar de que la planta necesita ser } \\
\text { regada. }\end{array}$ \\
\hline $\begin{array}{l}\text { 16.- Centre } \\
\text { Escolar San } \\
\text { Francisco } \\
\text { (Barcelona) }\end{array}$ & $\begin{array}{l}\text { 1.-Gimnasio urbano } \\
\text { (ODS 7) } \\
\text { 2.-ODS y comercio } \\
\text { de barrio (ODS 17) } \\
\text { 3.-Solas y seguras } \\
\text { (ODS 5) } \\
\text { 4.-Tercera edad } \\
\text { activa (ODS } 3 \text { ) }\end{array}$ & $\begin{array}{l}\text { 1.-Conseguir energía a través de los gimnasios, mediante } \\
\text { manivelas. } \\
\text { 2.-Reactivar la economía local del barrio Sant Martí de Provençals } \\
\text { mediante una app que alíe las tiendas. } \\
\text { 3.-Aplicación que localice a gente cerca de ti dispuesta a ayudarte o } \\
\text { desarrollar una pulsera que al pulsar ciertas veces llame a la policía. } \\
\text { 4.-Promover el deporte y dar ayudas económicas en proporción al } \\
\text { deporte realizado. }\end{array}$ \\
\hline $\begin{array}{l}\text { 17.- FEDAC Salt } \\
\text { (Girona) }\end{array}$ & $\begin{array}{l}\text { 1.-Optimizar energía } \\
\text { en las aulas (ODS } 4 \text { y } \\
\text { 11) } \\
\text { 2.-Zonas saludables } \\
\text { (ODS 3) }\end{array}$ & $\begin{array}{l}\text { 1.-Ahorrar energía mediante control de temperatura y luz. } \\
\text { Kit de arduino, Tinkercad, Chromeduino } 2 \text {. } \\
\text { 2.-Contabilizar la cantidad de personas que hay en un local o zona } \\
\text { comercial. Kit de arduino, Tinkercad, Chromeduino } 2 \text {. }\end{array}$ \\
\hline $\begin{array}{l}\text { 18.- Institut Maria } \\
\text { Rúbies (Lleida) }\end{array}$ & $\begin{array}{l}\text { 1.-Watercounter } \\
\text { (ODS 6) } \\
\text { 2.-Green 'nd Sunny } \\
\text { Roofies (ODS } 7 \text { y } 11 \text { ) }\end{array}$ & $\begin{array}{l}\text { 1.-Diseñar un prototipo de contador de agua con avisador de luz. } \\
\text { 2.-Hacer una maqueta de una casa con un tejado verde (sensor de } \\
\text { humedad) y unas placas solares (térmicas para calentar el agua) y } \\
\text { fotovoltaicas (para generar electricidad). }\end{array}$ \\
\hline
\end{tabular}

En cuanto a la implicación de los voluntarios en la mentoría de los proyectos es importante recalcar que todo el proceso se tuvo que realizar de forma telemática, debido a que el Covid-19 impidió que se pudieran visitar presencialmente los centros. Esto ha permitido por un lado abrir el proyecto de mentoría a los centros participantes de Cataluña, asignando mentores a estos, y no circunscribirse sólo al área de influencia de la universidad, en Madrid, pero a la vez ha sido comentado por los voluntarios como uno de los aspectos que han impedido tener una experiencia más completa, por la falta de contacto directo con los alumnos de secundaria. De hecho, la mentoría ha consistido principalmente en el contacto por correo electrónico con los profesores asignados, y no se han aprovechado, excepto algún caso, otras opciones como las conexiones virtuales de los mentores y las clases de los centros. 
Por otro lado, se han dado dos situaciones en relación con la respuesta de los profesores. En primer lugar, un grupo de mentores ha podido conocer los proyectos que se estaban realizando en las aulas. Su función principal se ha basado en responder dudas y problemas sobre las tecnologías que utilizaban para desarrollar los proyectos, tanto a profesores como a los propios alumnos. Incluso algunos voluntarios han tenido que investigar con mayor profundidad de manera autónoma sobre las tecnologías para poder resolver las dudas o incluso para realizar charlas para el alumnado. En uno de los casos dicha charla al final no pudo coordinarse para impartirse en línea, por lo que el voluntario creó un manual para lograr paso a paso programar lo necesario para completar el proyecto de los alumnos. Un mentor señala que tras la finalización de la actividad de este año, ha establecido una futura colaboración con la profesora asignada, para desarrollar un proyecto conjunto y dar alguna charla vocacional o según los intereses de los estudiantes en el centro.

Un segundo grupo de mentores señalan la dificultad que les ha supuesto conseguir respuesta por parte de los profesores asignados, lo que ha impedido que pudieran tener un rol más activo en el desarrollo de los proyectos. A este respecto, desde la Coordinación del proyecto CTM señalan que esta edición ha sido la más complicada hasta la fecha y que si bien normalmente a partir de febrero, los profesores solían comenzar con sus proyectos STEM, este año, las dificultades han provocado que la mayoría lo retrasasen varios meses y abordasen aspectos más sencillos para poder acabar los proyectos a final de curso.

Los mentores, por su parte, al finalizar el proyecto, han realizado informes de diagnóstico de necesidades formativas no cubiertas por el programa de formación a profesores de CTM, así como propuestas de mejora del proyecto y de la participación de los mentores. Han resaltado como necesidades formativas el diseño de formaciones con un mayor nivel para tener un mejor dominio de las tecnologías. Consideran que, para cualquier proyecto mínimamente interesante, es necesario un nivel más avanzado. Por ello, varias de las propuestas se dirigen a una oferta más personalizada según el grupo de participantes. Es decir, se podría formar a un nivel más introductorio a los profesores y a uno más avanzado a los mentores, ya que son los que tendrán que resolver dudas más complicadas y los que ya disponen de un nivel básico debido a sus estudios universitarios.

Las propuestas señaladas para la mejora del proyecto incluyen principalmente una mayor implicación por parte del profesorado o establecer un mínimo de reuniones entre mentor y profesor. Además, si se dan formaciones más avanzadas a los mentores, se podrían abordar proyectos más avanzados y que los alumnos se sintieran más realizados, sobre todo los de cursos superiores. En cualquier caso, la mayoría de los mentores han valorado muy positivamente las formaciones recibidas al inicio del proyecto por parte de CTM.

Por otro lado, cabe destacar que a pesar de todas las dificultades de este año y de la imposibilidad de realizar acciones presencialmente, la mayor parte de los mentores coincide en que la experiencia aprendizajeservicio ha sido muy positiva a nivel personal porque han visto cómo los alumnos a los que ayudaban se implicaban pensando ideas y avanzaban en los diferentes proyectos. Señalan como muy enriquecedor el poder ponerse en el lugar del alumno y poder realizar materiales o explicar las dudas de forma más intuitiva y clara. También señalan el interés por las formaciones recibidas, en las que pudieron ver cómo aterrizar muchos de sus conocimientos STEM en la enseñanza a otros niveles, en proyectos relacionados con los ODS, y con metodologías no tradicionales.

En cuanto a la evaluación de los mentores, estaba previsto realizar una autoevaluación y heteroevaluación del proceso, para lo cual se han diseñado herramientas para valorar el ApS por parte de los diferentes agentes involucrados. La autoevaluación ha sido realizada en los informes finales entregados, en los que los mentores han reflejado su percepción de los aprendizajes realizados durante las formaciones y durante 
la realización del proyecto. La heteroevaluación estaba planificada involucrando a diferentes agentes: profesores de los centros, alumnos escolares y equipo UPM-CTM, si bien este curso sólo podrán ser evaluados por los profesores de los centros y el equipo CTM, puesto que no todos han podido relacionarse de forma directa con los alumnos. Esta evaluación será llevada a cabo tras el evento final de presentación de proyectos. En dicho evento, se contará con la participación de dos mentores, que se han ofrecido a actuar como dinamizadores, a pesar de haber terminado ya la actividad. Este evento final se celebrará de forma virtual, y en ediciones anteriores y presenciales, se convertía en el momento estelar de CTM, cuando los escolares presentaban sus proyectos al resto y celebraban los retos abordados y los logros.

Desde el equipo CTM y UPM, se quiere destacar que este proyecto de innovación educativa ha arrancado en circunstancias difíciles para todas las partes involucradas, pues tanto mentores universitarios como profesores y alumnos escolares están inmersos en una docencia adaptada a la realidad de la pandemia, que impide realizar el proyecto CTM como se venía haciendo en ediciones anteriores (incluyendo la posibilidad de asistir a las aulas, interactuar con los alumnos y participar en el evento final presencialmente). A pesar de ello, los mentores han estado involucrados, participando y adaptándose a los plazos cambiantes.

\section{Conclusiones}

La participación de estudiantes universitarios en proyectos de Aprendizaje-Servicio puede resultar en mejores resultados de aprendizaje, debido a su carácter aplicado y la motivación por realizar un servicio a la comunidad (Rodríguez Gallego, 2014, Novak, Markey \& Allen, 2007). Por ello, la colaboración de alumnos de la UPM con organizadores de CTM y centros escolares de la Comunidad de Madrid y Cataluña se vislumbra como una experiencia positiva para todos los implicados. Por un lado, los estudiantes de la universidad consolidan contenidos propios de sus planes de estudio, pudiendo aplicarlos en proyectos reales que se llevan a cabo para mejorar el entorno inmediato del alumnado de los centros educativos implicados. Además, desarrollan otra serie de competencias relacionadas con el ámbito ético y social, que complementan su formación como futuros ingenieros.

Por otro lado, se fomentan las competencias científico-tecnológicas de alumnos de educación primaria y secundaria, cuyos docentes pueden apoyarse en los buenos conocimientos técnicos de estudiantes de ingenierías para poder llevar a cabo con éxito proyectos STEM que impliquen un impacto positivo en su comunidad. Todos los proyectos planteados hasta ahora con esta iniciativa van dirigidos a solucionar situaciones o circunstancias de actualidad y que preocupan a los escolares. Que éstos puedan proponer ellos mismos, con una formación tecnológica, soluciones viables a problemas reales que ellos mismos perciben, supone una buena oportunidad para motivarlos por el estudio de materias STEM, que contribuyen a su desarrollo personal, pero también social, por lo que revierten de forma útil a la comunidad. Por otro lado, se da respuesta a variados ODS, contribuyendo a su desarrollo. Asimismo, la cercanía, en edad y mentalidad, de los estudiantes universitarios con los alumnos de centros escolares, facilita un mayor intercambio y fomenta en mayor medida la motivación de los alumnos, implicándoles en el aprendizaje y desarrollo de competencias del ámbito científico-tecnológico. Esta relación entre distintos niveles educativos puede contribuir a objetivos tan relevantes como el desarrollo de competencias STEM y de los ODS, pero también un mayor contacto real con el mundo académico que facilite la elección vocacional de los alumnos de Secundaria.

También el propio proyecto CTM de la fundación Empieza Por Educar se beneficia de las sinergias creadas al recibir retroalimentación de los mentores universitarios participantes, que contribuirá a mejorar la experiencia en el futuro, y que se difundirá en foros educativos y científicos.

No obstante, los logros derivados del proyecto en este curso académico se han visto limitados por las circunstancias actuales de la pandemia, que ha restringido en gran medida las posibilidades de participación 
e interacción personal. Al perderse la relación directa entre mentores y centros (profesores y alumnos), el proyecto ha podido resultar menos satisfactorio para los universitarios. Además, algunos profesores de secundaria se encontraban a su vez tan desbordados por su labor docente adaptada al protocolo COVID, que no han contado realmente con los mentores asignados. Éstos han manifestado aún así su interés y motivación por la experiencia de ApS, adaptándose a la difícil situación.

La relación generada entre los agentes organizadores y los participantes en el proyecto de ApS tiene un gran potencial para seguir creciendo y contribuyendo, tanto al aprendizaje en competencias éticas, sociales y STEM de los alumnos escolares y universitarios, como al servicio que los proyectos ofrecen a la comunidad. La actividad, desde su oferta en el catálogo de actividades acreditables, ha tenido buena acogida por parte de los estudiantes universitarios, a pesar de las limitaciones ya descritas. Para cursos académicos posteriores se espera poder continuar con este proyecto y, cuando se pueda retomar en su versión presencial, contar con una metodología y sinergias ya consolidadas.

\section{Referencias}

BRINGLE, R.G., BROWN, L.A., HAHN y T.W., STUDER, M. (2019). "Pedagogies and civic programs to develop competencies for democratic culture and civic learning outcomes" en $\% R L G y Q$ vol. 71, issue 3, p. 27-43. <https://doi.org/10.13042/Bordon.2019.72003>

FROLOV, A.V. (2010). "The Role of STEM education in the "new economy" of the USA" en 4 XHMRQV $R \square$ KKHQHE $\square$ HFRQR $\backslash \square$ vol. 4, issue 16, p. 80-90.

GARCÍA LASO, A., NUÑEZ VARELA, E., MARTÍN SÁNCHEZ, D. A., RODRÍGUEZ RAMA, J. A., y COSTAFREDA MUSTELIER, J. L. (2019). "Aprendizaje-Servicio (ApS) como metodología para alcanzar los Objetivos de Desarrollo Sostenible (ODS)”. En: ,; $\square$ \&RQJUHR , QUAQDFIRQDO VREUH \$ SUHQC]DNHI

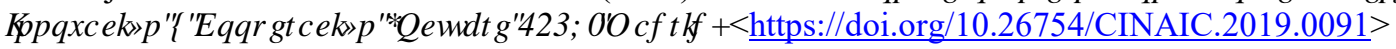

LÓPEZ-DE-ARANA PRADO, E., ARAMBURUZABALA HIGUERA, P. y OPAZO CARVAJAL, H. (2020). "Diseño y validación de un cuestionario para la autoevaluación de experiencias de aprendizajeservicio universitario" en (GXFDFlyQ ; ; $\square$ vol. 23(1), p. 319-347. $<$ https://doi.org/10.5944/educXX1.23834>

MORALES-ALONSO, G., PABLO-LERCHUNDI, I., y NÚÑEZ-DEL-RÍO, M. C. (2016). "Entrepreneurial intention of engineering students and associated influence of contextual factors/Intención emprendedora de los estudiantes de ingeniería e influencia de factores contextuales" en 5HLLD GH 3 UFRRJ tD6RFIDQvol. 31(1), p. 75-108. < https://doi.org/10.1080/02134748.2015.1101314>

NOVAK, J. M., MARKEY, V., y ALLEN, M. (2007). "Evaluating cognitive outcomes of service learning in higher education: A meta-analysis" en \&RP P XQIFDURQ 5HMDFK 5HSRLW, vol. 24 (2), p. 149-157. $<$ https://doi.org/10.1080/08824090701304881>ם

RODRÍGUEZ GALLEGO, M. R. (2014). "El Aprendizaje-Servicio como estrategia metodológica en la Universidad" en 5HIMD \&RPSOWAQM GH (GXFDFyQD vol. 25 (1), p. 95-113. $<$ https://doi.org/10.5209/rev_RCED.2014.v25.n1.41157>

SHATUNOVA, O., ANISIMOVA, T., SABIROVA, F., \& KALIMULLINA, O. (2019). "STEAM as an Innovative Educational Technology" en - RXLQDORILRFIDO6WGHHL( GXFDURQ5 HMDFK, vol. 10 (2), p. 131144.

SMITH-TOLKEN, A.R. Y MCKAY, M. (2019). "To be or not to be. Service-learning in a higher education institution" en \%RLy Qlvol. 71(3), p. 205-220. <https://doi.org/10.13042/Bordon.2019.72004> 